\title{
INDIGENOUS USES AND PHYTOCHEMICAL CONTENTS OF PLANTS USED IN THE TREATMENT OF MENSTRUAL DISORDERS AND AFTER- CHILD BIRTH PROBLEMS IN ABEOKUTA SOUTH LOCAL GOVERNMENT AREA OF OGUN STATE, NIGERIA
}

\author{
*Kadiri M, Ojewumi A.W, Onatade T. N \\ Department of Pure and Applied Botany, Federal University of Agriculture, Abeokuta, Ogun State Nigeria \\ *Corresponding Author's E-mail: mukaila kadiri@yahoo.com
}

Received 26 Feb 2015; Review Completed 05 April 2015; Accepted 16 April 2015, Available online 15 May 2015

\begin{abstract}
A survey of plants used for the treatment of menstrual disorders and after-child birth problems was conducted in Abeokuta South Local Government. Hundred (100) questionnaires were administered using multi stage sampling method on traditional herbal practitioners. Ethnobotanical information such as, plants and plant parts used, methods of extraction used and mode of administration of the herbal preparations of these plants were requested. Also, phytochemical contents of the most frequently mentioned plants were determined. Data were subjected to Analysis of variance (ANOVA) with probability set at $\mathrm{p}<0.05$ and descriptive statistics. Results revealed that a total of fifty-six (56) plants belonging to 37 families were mentioned. The most frequently mentioned families are Euphorbiaceae, Leguminoceae, Anacardiaceae, Apocynaceae, Araceae and Combretaceae. Sesamum indicum, Dioclea sarmentosa, Clausena anisata, Anogeissus leiocarpus, Alafia barteri, Tetrapleura tetraptera, Daniella oliveri, Lannea egregia and Alstonia boonei were the most frequently mentioned plants used in the treatment of menstrual disorders and after-childbirth problems. Leaves (34\%), fruits (7\%), flowers (3\%), tubers (2\%), stem-barks $(28 \%)$, seeds $(11 \%)$, roots and barks $(2 \%)$ and roots $(13 \%)$ were the plants reported being used for the remedy of these disorders. Decoction (54\%), squeezing (9\%), grinding/squeezing (14\%), paste (4\%), exudation (4\%), cooking (4\%), soaking/ decoction/infusion (4\%), and heating to ashes (4\%) were the methods of extracting the bioactive principles of the plants using water $(79 \%)$ as major solvent. Significant difference $(\mathrm{P}<0.05)$ was observed in the quantities of tannins recorded in the leaves of Sesamun indicum, Dioclea sarmentosa, Clausena anisata, Anogeissus leiocarpus and Alafia barteri. Similar observations were found in the quantities of saponnins, alkaloids, flavonoids and phenol. Highest values of tannins $(0.32 \mathrm{mg} / \mathrm{g})$, saponnins $(1.07 \mathrm{mg} / \mathrm{g})$, alkaloids $(5.16 \mathrm{mg} / \mathrm{g})$, flavonoids $(3.12 \mathrm{mg} / \mathrm{g})$ and phenol $(0.09 \mathrm{mg} / \mathrm{g})$ were determined in the leaves of Clausena anisata, Sesanum indicum, Dioclea sarmentosa and Alafia barteri. Across the roots of these plants, similar amount of tannins and saponnins were quantified. This observation varied significantly when compared with alkaloids, flavonoids and phenol quantified in the roots of plants. Highest tannins $(1.67 \mathrm{mg} / \mathrm{g})$ and saponnins $(3.33 \mathrm{mg} / \mathrm{g})$ were recorded in the roots of Dioclea sarmentosa, alkaloids (4.33 mg/g) and flavonoid $(6.33 \mathrm{mg} / \mathrm{g})$ in Anogeissus leiocarpus while phenol (1.33 mg/g) was recorded in roots of Sesanum indicum.
\end{abstract}

Key words: Menstrual Disorders, Childbirth Problems, phytochemical contents, Traditional practitioners, Indigenous plants

\section{INTRODUCTION}

Menstrual disorder and after problems such as amenorrhea, dysmenorrhea, menorrhagia, oligomenorrhea, after child birth pains, achy muscles, constipation, hemorrhoids and sore breasts (Nitta et al., 2002) are among some of the major challenges in various maternity homes and hospitals in Nigeria since majority of people are poor -famers and middle- men who in their local communities are poorly served with modern health facilities even at their pregnancy state and could not afford the exorbitant prizes of modern drugs. Disturbances of menstruation, either actual or perceived, are the most common presenting complaint of adolescents attending gynecology clinics. Problems associated with menstruation actually affect $75 \%$ of adolescent females and are a leading cause of such (c) 2011-15, JDDT. All Rights Reserved visits to physicians (Hajaratu et al.,2014). Also, postpartum depression has been reported to adversely affect mothers, their newborn infants, their partners and the society. The prevalence of postpartum depression among mothers was $30.6 \%$ at an Edinburgh Postnatal Depression scale (EPDS). Ukaegbe et al., 2012.

To avert this health challenge, plants play significant roles during pregnancy, birth and postpartum care in many rural areas of the world most especially developing country such as Nigeria. This is because Nigerian ecosystems are naturally endowed with arrays of floristic composition of different plant forms and resources (Olajide, 2003), thus enabling them to 
increase interest in traditional practices of health care as a complement to biomedical health care and part of primary health care delivery system in Nigeria (Sheldon el al., 1997).

Numerous botanicals with medicinal properties suitable for control and management of women's health related conditions such as, menorrhea, birth control, pregnancy, birth (parturition), postpartum, lactation and infant care, have been documented for various ethnic groups.

The postpartum period is important in many cultures, and is regarded as a period of recovery and confinement ranging from 10 up to 45 days. In accordance with humoral medicine, pregnancy is described as a hot state. During this parturition, heat is lost and the woman comes into a state of excess cold, Confinement as a treatment such as staying inside and near heat, washing only with hot water, drinking hot drinks, eating hot food, steam bath and bathing and staying away from draughts have been reported as measures of managing the situation(Davis, 2001).

This study was carried out to identify and characterize botanicals used for management of menstrual disorders and after childbirth problems. Also, Phytochemicals contents of most prioritized plants were evaluated.

\section{MATERIALS AND METHODS}

\section{STUDY AREA}

Abeokuta is the capital of Ogun state and traditionally home of Egbas stratified into Abeokuta North and Abeokuta south Local Government Area. The Egbas have been traditionally divided into four (4) namely Egba Ake, Oke-Ona,Gbagura and Owu. Three types of religion are widely practiced by the people. The religion includes Christianity, Islam and traditional religion. The Christian religion is predominant (Adekunle and Oluwalana, 2000). Geographically, Abeokuta lies on latitude $7^{\circ} 15 \mathrm{~N}$ and longitude $3^{\circ} 25 \mathrm{E}$. The town is about $81 \mathrm{~km}$ south- west of Ibadan, the Oyo State capital and 106km North of Lagos, former Nigerian capital city. Abeokuta has humid weather with an average temperature of about $27.4^{\circ} \mathrm{C}$ and an annual rainfall of $128 \mathrm{~cm}$ in the southern part of the city to $105 \mathrm{~cm}$ in Northern part. The Ogun river transverses through the town from the south to the western part.

The main occupation of the Egba people is farming, local textile,(Tie and dye), trading, pottery and industry.

\section{STUDY SITE}

A total of five (5) markets; Omida, Itoku, Adatan, Kuto and Panseke were visited. During the survey various shops of herbal practitioners were visited and the indigenous people were also interviewed to elicit information on the plants used for the treatment of menstrual disorders and after childbirth problems.

\section{Methodology of the study}

The study was carried out in the following stages:
Stage 1: Collection and review of published and unpublished literatures on plants used for treatment of menstrual disorder and after birth problems in Abeokuta, Ogun State, Nigeria

Stage 2: Questionnaire administration

\section{Sampling procedure and data collection}

A three stage design was adopted to collect data during this study.

Stage 1: Selection of Abeokuta south Local Government areas to represent primary collection unit;

Stage 2: Purposive selection of 5 markets in the selected Local Government Area

Stage 3: Random selection of 20 respondents (herbal practitioners comprising herbal sellers and herbal and traditional healers) in the Local Government

\section{Table 1: Sampling design}

\begin{tabular}{lc}
\hline Markets & Number of respondents selected \\
\hline Kuto & 20 \\
Adatan & 20 \\
Panseke & 20 \\
Itoku & 20 \\
Omida & 20 \\
Total population of the study & 100 \\
\hline
\end{tabular}

A total of 100 questionnaires administered were validated and reliability test of the questionnaire was also carried out.

Table 2: Reliability Statistics

\begin{tabular}{|c|c|}
\hline Cronbach's Alpha $^{\mathrm{a}}$ & Number of Items \\
\hline 0.77 & 20 \\
\hline
\end{tabular}

\section{Quantitative phytochemical screening of leaves and roots of most frequently mentioned plants}

Phytochemical contents such as tannins, saponnins, alkaloids, flavonoids and phenol of leaves and roots of the most frequently mentioned plant were carried out using methods of Ojewumi and Kadiri, 20014

\section{Statistical Analysis}

Data were subjected to Analysis of variance (ANOVA) and separation of means by Duncan's multiple ranges Test (DMRT) at $\mathrm{P}<0.05$.

\section{RESULTS}

Larger number of the respondents $71(72.4 \%)$ were females. Majority of the respondents (traditional practitioners) 44(44.45) were between the age 2140years while least of them $11(11.1 \%)$ were less than 21 years. Islam was the predominant religion of the respondents. Also, Majority of the respondents $(83.8 \%)$ acquired formal education (Table 3$)$. 
Fifty-six (60) plant species belonging to 37 families were collated out of which Euphorbiaceae, Leguminoasae, Anacardiaceae and Apocynaceae were the most frequently mentioned families. The most frequently mentioned plants were Sesamun indicum, Dioclea sarmentosa, Clausena anisata, Anogeissus leiocarpus and Alafia barteri(Table 4). The life forms of plants collated during this study ranged from trees to herbs out of which trees were the most mentioned (Table 5).

Largest number of traditional practitioners 42(43.8\%) were herb sellers with more than ten (10) years work experience in the business. They obtained their herbal knowledge mainly by training as denoted by 51.1 percent. 82.2 percent of them claimed to treat either of disorders on weekly basis. Fifty four percent (54\%) of the herbal practitioners reported that the plants were cultivated at home gardens (Table 7).

The plant collated during this survey were reportedly being used indifferent forms such as dry (28\%), fresh $(65 \%)$ and combination of dry and fresh (7\%) depending on the availability of the plants and severity of the diseases. Several plant parts such as leaves $(34 \%)$, fruits $(7 \%)$, flower $(3 \%)$, tuber ( $2 \%)$, stembark $(28 \%)$, seeds $(11 \%)$, roots and barks $(2 \%)$ and roots $(13 \%)$ were reported (Figure 2).Decoction(54\%), squeezing (9\%), grinding/squeezing $(14 \%)$, paste $(4 \%)$, exudation (4\%), cooking (4\%), soaking/ decoction/infusion (4\%), and heating to ashes $(4 \%)$ were the methods of extracting the bioactive principles of the plants using water (79\%) as major solvent ( Figure 3 and 4). Preparations from these plants were reported to be taken 2-3times daily (Figure 5).

Largest number of the traditional practitioners indicated that most of the plants used were cultivated in their home gardens $(55.1 \%)$, followed by forest plants $(26.5 \%)$ while the least of them indicated swampy areas $(4.1 \%)$ as the sources of the plants used as remedies for menstrual disorders and after birth problems( Table 7).

Table 8 revealed the mean values of phytochemical contents of the leaves of plant commonly used for treatment of menstrual disorders and after childbirth problems in Abeokuta south Local Government Local Area, Abeokuta. There was significant difference $(\mathrm{P}<0.05)$ in the quantities of tannins recorded in some of the leaves of Sesamun indicum, Dioclea sarmentosa, Clausena anisata, Anogeissus leiocarpus and Alafia barteri. Similar observations were recorded in the quantities of saponnins, alkaloids, flavonoids and phenol. Also, except sesanum indicum, no significant $(\mathrm{P}>0.0)$ amount of saponnins wasa recorded in Dioclea sarmentosa, Clausena anisata, Anogeissus leiocarpus and Alafia barteri. Highest values of tannins $(0.32 \mathrm{mg} / \mathrm{g}), \quad$ saponnins $\quad(1.07 \mathrm{mg} / \mathrm{g}), \quad$ alkaloids $(5.16 \mathrm{mg} / \mathrm{g})$, flavonoids $(3.12 \mathrm{mg} / \mathrm{g})$ and phenol $(0.09$ $\mathrm{mg} / \mathrm{g}$ ) were determined in the leaves of Clausena anisata, Sesanum indicum, Dioclea sarmentosa, Alafia barteri (Table 8 ).

Across the roots of these plants, similar amounts of tannins and saponnins were quantified. This observation varied significantly when compared with alkaloids, flavonoids and phenol quantified in the roots of plants. Highest tannins $(1.67 \mathrm{mg} / \mathrm{g})$ and saponnins $(3.33 \mathrm{mg} / \mathrm{g})$ were recorded in the roots of Dioclea sarmentosa, alkaloids (4.33 mg/g) and flavonoid (6.33 $\mathrm{mg} / \mathrm{g}$ ) in Anogeissus leiocarpus while phenol (1.33 $\mathrm{mg} / \mathrm{g}$ ) was recorded in roots of Sesanum indicum (Figure 9)

Table 3: Demographic profile of respondents of the study area

\begin{tabular}{|l|l|l|l|}
\hline Variables & Frequency & Percentage frequency & Mode \\
\hline Sex & & & \\
\hline Male & 27 & 27.6 & 72.4 \\
\hline Female & 71 & 72.4 & \\
\hline Age (years) & & & \\
\hline less than 21 & 11 & 11.1 & 44.4 \\
\hline $21-40$ & 44 & 44.4 & \\
\hline $41-60$ & 29 & 29.3 & \\
\hline More than 60 & 15 & 15.2 & \\
\hline Religion & & & 60.8 \\
\hline Christianity & 15 & 15.5 & \\
\hline Islam & 59 & 60.8 & 34.3 \\
\hline Traditional & 22 & 22.7 & \\
\hline Educational status & & & \\
\hline Primary & 34 & 34.3 & \\
\hline Secondary & 33 & 33.3 & \\
\hline Tertiary & 16 & 16.2 & \\
\hline No formal education & 16 & 16.2 & \\
\hline
\end{tabular}


Table 4: Plants commonly used for control and management of menstrual disorder and after birth problems in Abeokuta south Local Government Local Area, Abeokuta, Ogun State.

\begin{tabular}{|c|c|c|c|c|c|}
\hline Scientific name & Local name & Common name & Family & Habitat & Part used \\
\hline $\begin{array}{l}\text { Abelmoschus } \\
\text { esculentus }\end{array}$ & Ila pupa & Red okra & Malvaceae & Shrubs & Seeds \\
\hline $\begin{array}{l}\text { Aframomum } \\
\text { melegueta }\end{array}$ & Atare & Alligator peper & Zingiberaceae & Herbs & Seeds \\
\hline Alafia barteri & Agbari etu & Guinea fowl's crest & Apocynaceae & Climbing shrubs & Leaves \\
\hline Allium ascalonicum & Alubosa elewe & Shallot & Liliaceae & Herbs & Leaves \\
\hline Alstonia boonei & Awun & Stool wood & Apocynaceae & Tree & Stem barks \\
\hline $\begin{array}{l}\text { Anogeissus } \\
\text { leiocarpus }\end{array}$ & Orin dudu & African birch & Combretaceae & Tree & Stem bark \\
\hline Aristolochia repens & Akogu & Dutchman's pipe & Aristolochiaceae & Herbs & Leaves \\
\hline Basella alba & Amunututu & Indian spinach & Basellaceae & Herbs & Leaves \\
\hline Bridelia ferruginea & Ira & Bridelia & Euphorbiaceae & Tree & Stem bark \\
\hline Calotropis procera & Bomu bomu & Giant milk weed & Asclepidiaceae & Shrubs & Leaves \\
\hline Capsicum frutescens & Ata ijosi & Hot pepper & Solanaceae & Herbs or Shrubs & Seeds \\
\hline Carica papaya & Ibepe & Pawpaw & Caricaceae & Shrubs & Roots \\
\hline Ceiba petandra & Araba & Silk cotton tree & Bombacaceae & Tree & Roots \\
\hline $\begin{array}{l}\text { Cissampelos } \\
\text { mucronata }\end{array}$ & Jenjoko/Jokoje & Ivy vine & Menispermaceae & Climbers herbs & Leaves \\
\hline $\begin{array}{l}\text { Cissus } \\
\text { quadrangularis }\end{array}$ & Olowomefa & $\begin{array}{l}\text { Edible stemmed } \\
\text { vine }\end{array}$ & Vitaceae & Herbs & Stem barks \\
\hline Citrus medica & $\begin{array}{l}\text { Osan } \\
\text { ijaganyin }\end{array}$ & Citron & Rutaceae & Shrubs or Tree & Fruits \\
\hline Clausena anisata & Ata pari obuko & Horse wood tree & Rutaceae & Tree & Root \\
\hline Cocos nucifera & Agbon & Coconut palm & Arecaceae & Tree & Fruits \\
\hline Costus afer & Teteregun & $\begin{array}{l}\text { Common ginger } \\
\text { lily }\end{array}$ & Costaceae & Herbs & Seeds \\
\hline Croton lobatus & Eru alamo & Garden croton & Euphorbiaceae & Herbs & Fruits \\
\hline Culcasia scandens & Agunmonu & Climbing arum & Araceae & Climbing herbs & Leaves \\
\hline Daniella oliveri & Iya & Balsam tree & Leguminoceae & Tree & Stem barks \\
\hline $\begin{array}{l}\text { Dichapetalum } \\
\text { toxicarium }\end{array}$ & Ewo & $\begin{array}{l}\text { West African rats } \\
\text { bane }\end{array}$ & Chailleriaceae & Shrubs & Roots \\
\hline Dioclea sarmentosa & Dasha & Sea beans & Leguminosae & Climbers herbs & Leaves \\
\hline Dioclea sarmentosa & Dasa & Sea beans & Leguminoasae & Herbs & Leaves \\
\hline Euphorbia hirta & Emi-ile & Asthma-weed & Euphorbiaceae & Herbs & Leaves \\
\hline Garcinia kola & Orogbo & Bitter kola & Guttiferae & Tree & Stem barks \\
\hline Hibicus acetosella & Akese & $\begin{array}{l}\text { African rose } \\
\text { mallow }\end{array}$ & Malvaceae & Shrubs & Leaves \\
\hline Hunteria umbellata & Abeere & Demouan & Apocynaceae & Tree & $\begin{array}{l}\text { Roots and } \\
\text { barks }\end{array}$ \\
\hline Irvingia gabonensis & Oro & African mango & Irvingiaceae & Tree & Stem barks \\
\hline Jatropha curcas & Botuje & Physic nut & Euphorbiaceae & Shrubs & Leaves \\
\hline Jatropha gossypifolia & Botuje pupa & Wild cassava & Ephorbiaceae & Shrubs & Leaves \\
\hline Lannea egregia & Ekudan & False marula & Anacardaceae & Tree & Stem bark \\
\hline Lannea egregia & Epo ekudan & Woodier wood & Anacardiaceae & Tree & Stem bark \\
\hline Lawsonia inermis & Laali & Henna plant & Lytheraceae & Tree & Flowers \\
\hline Lophira lanceolata & Paran pupa & $\begin{array}{l}\text { Dwarf red } \\
\text { ironwood }\end{array}$ & Ochnaceae & Tree & Stem barks \\
\hline Mangifera indica & Mangoro & Mango & Anacardiaceae & Tree & Fruits \\
\hline Momordica charantia & Ejirin & Bitter gourd & Cucurbitaceae & Herb & Leaves \\
\hline Morus alba & Aye & White mulberrry & Moraceae & Tree & Stem barks \\
\hline Ocimum basilicum & Efinrin wewe & Sweet basil & Labiateae & Herb & Leaves \\
\hline Olax subscopioidea & Ifon & Olax & Olacaceae & Shrubs or Tree & Roots \\
\hline Parinari spp & Abeere & Hissing tree & Rosaceae & Tree & Seeds \\
\hline $\begin{array}{l}\text { Phyllanthus } \\
\text { muellerianus }\end{array}$ & Asasa & Leafflower & Euphorbiaceae & Shrubs or Herbs & Stem barks \\
\hline $\begin{array}{l}\text { Piliostigma } \\
\text { thonningii }\end{array}$ & Abafe & Kargo & Caesalpiniaceae & Tree & Stem barks \\
\hline
\end{tabular}




\begin{tabular}{|l|l|l|l|l|l|}
\hline Piper guineense & Iyere & Black pepper & Piperaceae & Shrubs or Herbs & Leaves \\
\hline Piper guineense & Iyere & Black pepper & Piperaceae & Herbs & Stem barks \\
\hline Pistia stratiotes & Oju oro & Water lettuce & Araceae & Herbs & Leaves \\
\hline Plumbago zeylanica & Inabiri & Ceylon leadwort & Plumbaginaceae & Shrubs & Roots \\
\hline Pterocarpus osun & Osun & Blood wood & Leguminosae & Tree & Leaves \\
\hline Rauvolfia vomitoria & Asofeyeje & Serpent wood & Apocynaceae & Shrubs or Tree & Roots \\
\hline Scleria racemosa & Labelabe tutu & Sword grass & Cyperaceae & Herbs & Roots \\
\hline Senna alata & Asuniwon & Craw-craw plant & Caesalpinaceae & Shrubs & Flowers \\
\hline Sesamum indicum & Ekuku & Sesame & Pedaliaceae & Herbs & Leaves \\
\hline Sorghum bicolor & Poroporo & Sorghum & Poaceae & Herbs & Seeds \\
\hline Spondias mombin & Ikika/Iyeye & Yellow mombin & Anacardiaceae & Tree & Stem barks \\
\hline Stephania abyssinica & Gbejedi & Menispermaceae & Climbing shrubs & Leaves \\
\hline $\begin{array}{l}\text { Pentaclethra } \\
\text { macrophylla }\end{array}$ & Apara & African oil bean & Leguminosae & Tree & Leaves \\
\hline $\begin{array}{l}\text { Terminalia } \\
\text { avicennioides }\end{array}$ & Idin & Bauche & Combretaceae & Tree & Stem-barks \\
\hline $\begin{array}{l}\text { Tetrapleura } \\
\text { tetraptera }\end{array}$ & Aidan & India laburnum & Leguminoceae & Tree & Seeds \\
\hline $\begin{array}{l}\text { Xanthosoma } \\
\text { sagittifolium }\end{array}$ & Koko funfun & White cocoyam & Araceae & Herbs & Tuber \\
\hline Xylopia aethiopica & Eru & Negro pepper & Annonaceae & Tree & Stem barks \\
\hline
\end{tabular}

Table 5: Life forms of plants collated in Abeokuta South Local Government

\begin{tabular}{|l|l|l|}
\hline Life forms & Frequency & Percentage frequency \\
\hline Trees & 24 & 40.0 \\
\hline Shrubs or tree & 3 & 5.0 \\
\hline Shrubs & 9 & 15.0 \\
\hline Herb or shrubs & 3 & 5.0 \\
\hline Herbs & 16 & 26.67 \\
\hline Climbing shrubs & 5 & 8.33 \\
\hline Total & 60 & \\
\hline
\end{tabular}

Table 6: Recipes used in the treatment of menstrual disorders after birth problems

\begin{tabular}{|c|c|c|c|c|}
\hline Diseases & Recipes & $\begin{array}{l}\text { Traditional } \\
\text { solvent of } \\
\text { choice }\end{array}$ & $\begin{array}{l}\text { Method of } \\
\text { preparation }\end{array}$ & $\begin{array}{l}\text { Method of } \\
\text { administration }\end{array}$ \\
\hline \multicolumn{5}{|l|}{ Menstrual disorder } \\
\hline Painful menstruation & $\begin{array}{l}\text { Dioclea sarmentosa, Sesamum indicum, } \\
\text { Aloe barteri }\end{array}$ & Water & Decoction & $\begin{array}{l}\text { Take decoction 2-3 } \\
\text { times daily }\end{array}$ \\
\hline $\begin{array}{l}\text { Menorrhahia } \\
\text { (excessive menstrual } \\
\text { bleeding) }\end{array}$ & Dioclea sarmentosa, potash/alum & Water & Decoction & $\begin{array}{l}\text { Oral consumption of } \\
\text { decoction of } \\
\text { Leaves }\end{array}$ \\
\hline $\begin{array}{l}\text { Irregular menstrual } \\
\text { flow }\end{array}$ & $\begin{array}{l}\text { Jatropha gossypifolia and Dioclea } \\
\text { sarmentosa }\end{array}$ & Water & Squeezing & $\begin{array}{l}\text { Oral consumption of } \\
\text { juice from } \\
\text { squeezed leaves }\end{array}$ \\
\hline Heavy menstruation & Jatropha curcas & Water & Decoction & $\begin{array}{l}\text { Bath private part } \\
\text { with decoction }\end{array}$ \\
\hline $\begin{array}{l}\text { Foul smell of } \\
\text { menstrual discharges }\end{array}$ & $\begin{array}{l}\text { Sesamum indicum Pterocarpus osun, } \\
\text { Plumbago zeylanica, Piper guineensis, } \\
\text { Sulphur, Citrus medica, }\end{array}$ & Local gin & $\begin{array}{l}\text { Grinding/ } \\
\text { Squeezing }\end{array}$ & $\begin{array}{l}\text { Take orally (200- } \\
250 \mathrm{ml} \text { once daily) }\end{array}$ \\
\hline Ceased menstruation & Abelmoschus esculentus & Local gin & $\begin{array}{l}\text { Heating to } \\
\text { ashes }\end{array}$ & Take orally \\
\hline Painful menstruation & $\begin{array}{l}\text { Hibiscus acetosella, Dioclea } \\
\text { sarmentosa, Sesamum indicum }\end{array}$ & Water & Decoction & Take orally \\
\hline Abdominal pain & $\begin{array}{l}\text { Sorghum bicolor, Scleria racemosa, } \\
\text { Pterocarpus osun, Dichapetallum } \\
\text { toxicarium, potash, Pterocarpus osun, } \\
\text { Potash, Phyllanthus muellerianus, } \\
\text { Dioclea sarmentosa, Stephania }\end{array}$ & Water & Decoction & $\begin{array}{l}\text { Take orally every } \\
\text { night }\end{array}$ \\
\hline
\end{tabular}




\begin{tabular}{|c|c|c|c|c|}
\hline & sarmentosa small quantity of salt & & & \\
\hline \multirow[t]{2}{*}{ Dysmenorrhea } & Momordica charantia & Water & Decoction & Take orally \\
\hline & Lawsonia inermis & Water & $\begin{array}{l}\text { Paste of } \\
\text { leaves and } \\
\text { flower }\end{array}$ & Take orally \\
\hline $\begin{array}{l}\text { Blackish colour in } \\
\text { menses }\end{array}$ & Senna alata, Potash, Pap & Water & $\begin{array}{l}\text { Grind } \\
\text { flower to } \\
\text { powder }\end{array}$ & Take orally \\
\hline Menorrhagia & Ceiba petandra & Water & $\begin{array}{l}\text { Exudation } \\
\text { from the } \\
\text { trunk }\end{array}$ & Take as tonic \\
\hline $\begin{array}{l}\text { Premenstrual } \\
\text { syndrome }\end{array}$ & Cissus quadrangularis & Water & Cooking & Drink \\
\hline Black menses & Phyllanthus muellerianus & Water & Decoction & Drink \\
\hline $\begin{array}{l}\text { Antiperiodic } \\
\text { problem }\end{array}$ & Rauwolfia vomitoria & Water & Grinding & Drink \\
\hline \multicolumn{5}{|l|}{$\begin{array}{l}\text { After childbirth } \\
\text { problems }\end{array}$} \\
\hline Stomach pain & $\begin{array}{l}\text { Capsicum frutescens, Afromamomum } \\
\text { melegueta, Aframomum melegueta, } \\
\text { Capsicum frutescens Pterocarpus osun, } \\
\text { Sorghum bicolour, Lannea egregia } \\
\text { Daniellia oliveri, Lannea egregia, } \\
\text { Anogeissus leiocarpus, Piliostigma } \\
\text { thonningii }\end{array}$ & Local gin & $\begin{array}{l}\text { Soaking/ } \\
\text { Decoction }\end{array}$ & Taken orally \\
\hline $\begin{array}{l}\text { Bleeding after } \\
\text { delivery }\end{array}$ & $\begin{array}{l}\text { Basella alba, Cissampelos mucronata, } \\
\text { Stephania sarmentosa }\end{array}$ & Water & Decoction & $\begin{array}{l}\text { Drink 2-3 times } \\
\text { daily }\end{array}$ \\
\hline $\begin{array}{l}\text { Bleeding after } \\
\text { delivery }\end{array}$ & Spondias mombin, Irvingia gabonensis & $\begin{array}{l}\text { Fermented } \\
\text { maize water }\end{array}$ & Decoction & $\begin{array}{l}\text { Drink hot after } \\
\text { cooking }\end{array}$ \\
\hline Breast infection & $\begin{array}{l}\text { Olax subdcopioidea, Costus afer, Pistia } \\
\text { stratiotes, Ocimum basilicum, Xylopia } \\
\text { aethiopica, Tetrapleura tetraptera, } \\
\text { Anogeissus leiocarpus, Terminalia } \\
\text { avicennioiodes, Xylopia aethiopica }\end{array}$ & Local gin & Decoction & Bathe affected part \\
\hline $\begin{array}{l}\text { excessive uterine } \\
\text { bleeding }\end{array}$ & $\begin{array}{l}\text { Stephania sarmentosa, Cissampelos } \\
\text { mucronata, Basella alba }\end{array}$ & Water & Squeezing & Drink \\
\hline Pain in the breast & $\begin{array}{l}\text { Garcinia kola, Astonia boonei, Clausena } \\
\text { anisata, Culcasia scandens, Alafia } \\
\text { barteri }\end{array}$ & Water & Decoction & Drink \\
\hline Stomach ache & $\begin{array}{l}\text { Lophira lanceolata, Aristolochia repens, } \\
\text { Hunteria umbellate }\end{array}$ & Water & Decoction & Take orally \\
\hline $\begin{array}{l}\text { Bleeding after } \\
\text { delivery }\end{array}$ & $\begin{array}{l}\text { Irvingia gabonensis, Allium } \\
\text { ascalonicum, Gnetum africanum }\end{array}$ & Water & Decoction & Take orally \\
\hline Lactation problem & $\begin{array}{l}\text { Calotropis procera Carica papaya } \\
\text { Euphorbia hirta }\end{array}$ & Water & $\begin{array}{l}\text { Grind leaves } \\
\text { with local } \\
\text { soap/decocti } \\
\text { on/infusion }\end{array}$ & $\begin{array}{l}\text { Wash breast thrice } \\
\text { daily, oral } \\
\text { application }\end{array}$ \\
\hline
\end{tabular}


Table 7: Experience of herbal practices of the traditional practitioners of the study area

\begin{tabular}{|l|l|l|l|}
\hline Parameter & Frequency & Percentage frequency & Mode \\
\hline Herbal Practice specification & & & \\
\hline Herbalist & 5 & 5.2 & 43.8 \\
\hline Herb sellers & 42 & 43.8 & \\
\hline Traditional Medical practitioner & 13 & 13.5 & \\
\hline TMP/Herb sellers & 22 & 22.9 & \\
\hline Trained Medical Practitioner & 14 & 14.6 & \\
\hline Source of knowledge & & & 51.1 \\
\hline Ancestral & 26 & 28.3 & \\
\hline Training & 47 & 51.1 & \\
\hline Divination & 7 & 7.6 & \\
\hline Ancestral/ Training & 12 & 13.0 & \\
\hline Years of herbal practice experience & & & 41.4 \\
\hline $1-5$ years & 21 & 21.2 & 82.2 \\
\hline $6-10$ years & 37 & 37.4 & \\
\hline More than 10 years & 41 & 41.4 & \\
\hline Duration of treatment & & & \\
\hline 1 week & 60 & 82.2 & \\
\hline 2 weeks & 8 & 11.0 & 55.1 \\
\hline More than 2 weeks & 5 & 6.8 & \\
\hline Sources of plants & & & \\
\hline Forest alone & 26 & 26.5 & \\
\hline Cultivated at home garden & 54 & 55.1 & \\
\hline Swamp & 4 & 4.1 & \\
\hline Market & 7 & 7.1 & \\
\hline Forest and cultivated around house garden & 3 & 3.0 & \\
\hline Swamp and cultivated herb garden & 4 & 4.1 & \\
\hline Accompanied verbal instruction & & 2.1 & \\
\hline Yes & 2 & 97.9 & \\
\hline No & 92 & & \\
\hline
\end{tabular}

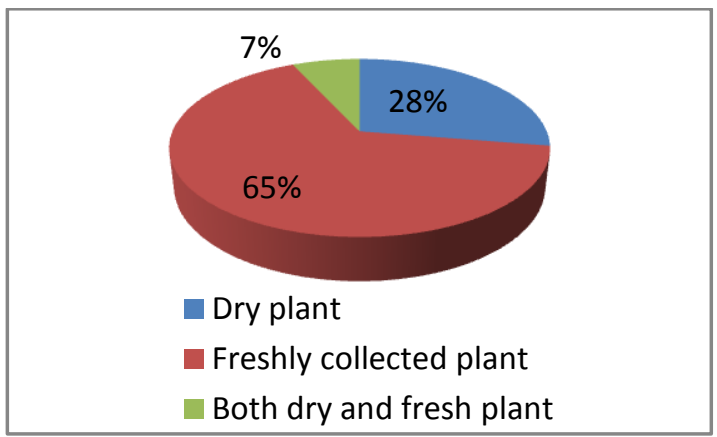

Figure 1: Forms of plants collection

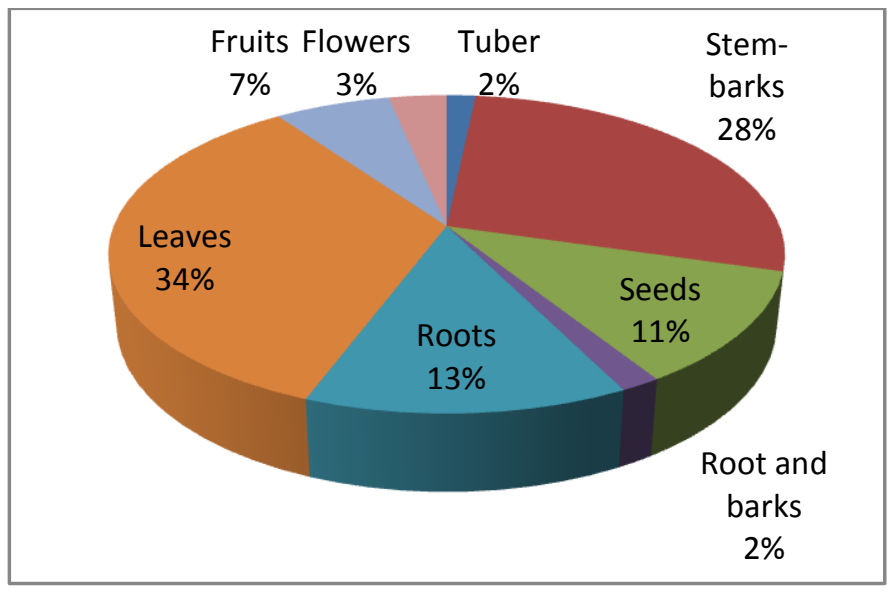

Figure 2: Plant parts used 


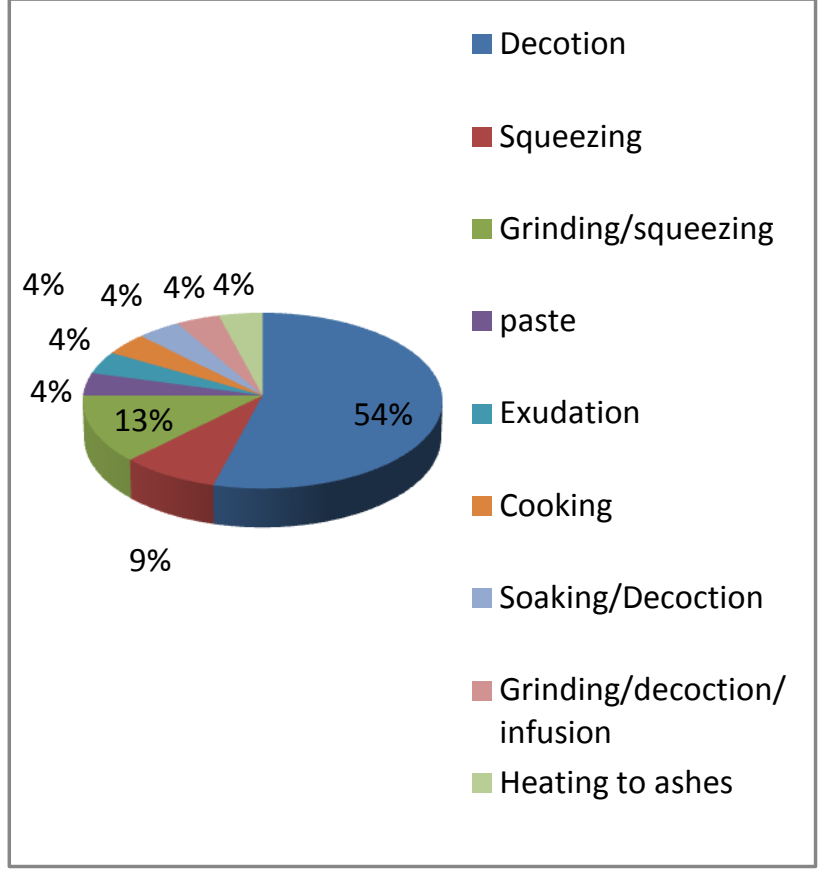

Figure 3: Methods of preparation

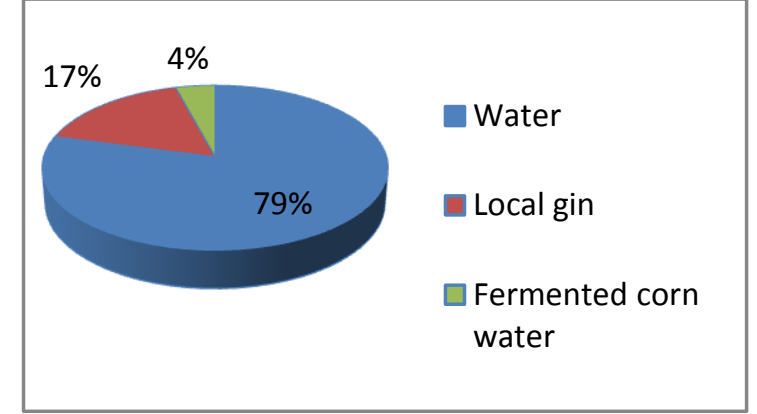

Figure 4: Solvents used

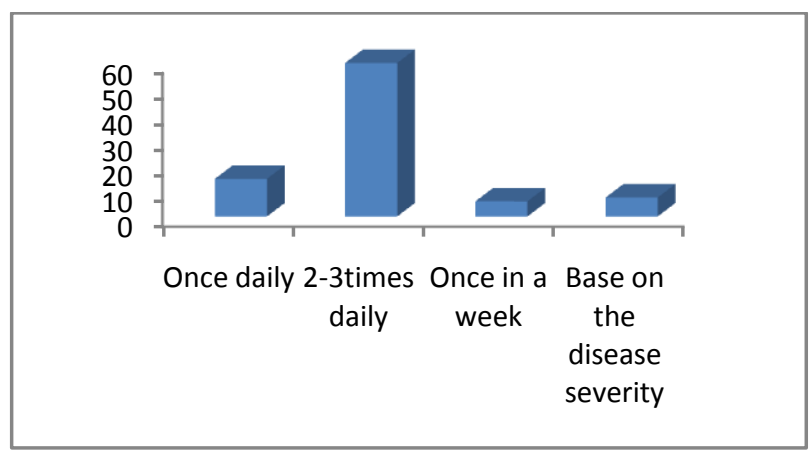

Figure 5: Frequency of usage of herbal preparation

Table 8: Mean values of phytochemical contents of the leaves of plant commonly used for treatment of menstrual disorders and after childbirth problems in Abeokuta south Local Government Local Area, Abeokuta, Ogun State.

\begin{tabular}{|l|l|l|l|l|l|}
\hline \multirow{2}{*}{ Plants/parts( leaves) } & \multicolumn{5}{|c|}{ Phytochemicals } \\
\cline { 2 - 6 } & $\begin{array}{l}\text { Tannins } \\
(\mathrm{mg} / \mathrm{g})\end{array}$ & $\begin{array}{l}\text { Saponins } \\
(\mathrm{mg} / \mathrm{g})\end{array}$ & $\begin{array}{l}\text { Alkaloids } \\
(\mathrm{mg} / \mathrm{g})\end{array}$ & $\begin{array}{l}\text { Flavanoids } \\
(\mathrm{mg} / \mathrm{g})\end{array}$ & $\begin{array}{l}\text { Phenol } \\
(\mathrm{mg} / \mathrm{g})\end{array}$ \\
\hline Sesanum indicum & $0.043 \pm 0.02^{\mathrm{c}}$ & $1.07 \pm 0.03^{\mathrm{a}}$ & $3.15 \pm 0.07^{\mathrm{d}}$ & $1.13 \pm 0.06^{\mathrm{d}}$ & $0.03 \pm 0.013^{\mathrm{b}}$ \\
\hline Dioclea sarmentosa & $0.23 \pm 0.01^{\mathrm{ab}}$ & $0.89 \pm 0.04^{\mathrm{b}}$ & $5.16 \pm 0.09^{\mathrm{a}}$ & $2.69 \pm 0.03^{\mathrm{b}}$ & $0.06 \pm 0.02^{\mathrm{b}}$ \\
\hline Clausena anisata & $0.32 \pm 0.03^{\mathrm{a}}$ & $0.72 \pm 0.03^{\mathrm{b}}$ & $3.79 \pm 0.26^{\mathrm{c}}$ & $1.86 \pm 0.03^{\mathrm{e}}$ & $0.05 \pm 0.01^{\mathrm{b}}$ \\
\hline Anogeissus leiocarpus & $0.14 \pm 0.07^{\mathrm{bc}}$ & $0.68 \pm 0.16^{\mathrm{b}}$ & $2.31 \pm 0.15^{\mathrm{e}}$ & $2.01 \pm 0.05^{\mathrm{c}}$ & $0.05 \pm 0.03^{\mathrm{ab}}$ \\
\hline Alafia barteri & $0.19 \pm 0.02^{\mathrm{ab}}$ & $0.69 \pm 0.12^{\mathrm{b}}$ & $4.12 \pm 0.29^{\mathrm{b}}$ & $3.12 \pm 0.02^{\mathrm{a}}$ & $0.09 \pm 0.02^{\mathrm{a}}$ \\
\hline
\end{tabular}

Means followed by the same letters on the same columns are not significantly different according to Duncan's Multiple Range Test at $p<0.05$.

Table 9: Quantitative phytochemical screening of the roots of plants mostly used in the treatment of menstrual disorders and after-childbirth problems.

\begin{tabular}{|l|l|l|l|l|l|}
\hline \multirow{2}{*}{ Plants/parts( roots) } & \multicolumn{5}{|c|}{ Phytochemicals } \\
\cline { 2 - 6 } & $\begin{array}{l}\text { Tannins } \\
(\mathrm{mg} / \mathrm{g})\end{array}$ & Saponins (mg/g) & $\begin{array}{l}\text { Alkaloids } \\
(\mathrm{mg} / \mathrm{g})\end{array}$ & $\begin{array}{l}\text { Flavanoids } \\
(\mathrm{mg} / \mathrm{g})\end{array}$ & Phenol (mg/g) \\
\hline Sesanum indicum & $0.74 \pm 0.74^{\mathrm{a}}$ & $1.33 \pm 0.33^{\mathrm{a}}$ & $2.33 \pm 0.33^{\mathrm{bc}}$ & $2.33 \pm 0.33^{\mathrm{c}}$ & $1.33 \pm 0.33^{\mathrm{a}}$ \\
\hline Dioclea sarmentosa & $1.67 \pm 0.33^{\mathrm{a}}$ & $1.67 \pm 0.67^{\mathrm{a}}$ & $1.67 \pm 0.34^{\mathrm{c}}$ & $2.01 \pm 1.01^{\mathrm{c}}$ & $1.00 \pm 0.01^{\mathrm{ab}}$ \\
\hline Clausena anisata $^{\mathrm{a}}$ & $1.01 \pm 0.58^{\mathrm{a}}$ & $2.67 \pm 1.20^{\mathrm{a}}$ & $2.33 \pm 0.33^{\mathrm{bc}}$ & $1.00 \pm 0.58^{\mathrm{c}}$ & $0.33 \pm 0.33^{\mathrm{b}}$ \\
\hline Anogeissus leiocarpus & $0.67 \pm 0.33^{\mathrm{a}}$ & $3.33 \pm 1.2^{\mathrm{a}}$ & $4.33 \pm 0.33^{\mathrm{a}}$ & $6.33 \pm 0.33^{\mathrm{a}}$ & $0.67 \pm 0.33^{\mathrm{ab}}$ \\
\hline Alafia barteri & $1.67 \pm 0.67^{\mathrm{a}}$ & $1.67 \pm 0.66^{\mathrm{a}}$ & $3.30 \pm 0.29^{\mathrm{b}}$ & $4.42 \pm 0.31^{\mathrm{b}}$ & $0.89 \pm 0.11^{\mathrm{ab}}$ \\
\hline
\end{tabular}

Means followed by the same letters on the same columns are not significantly different according to Duncan's Multiple Range Test at $p<0.05$.

\section{DISCUSSION}

The present study reveals that people of Abeokuta are well versed with the nature and natural resources around them despite the hash environmental factors being faced by these plants. These people in an attempt to get rid of various diseases such as menstrual disorder and after birth problems depend on plant products found in their immediate environment due to no or poor health facilities that were made available for them.

The observation that majority of the traditional practitioners claimed to treat either of the diseases on 


\section{Kadiri et al}

Journal of Drug Delivery \& Therapeutics. 2015; 5(3):33-42

weekly basis could be an indication that the diseases are very rampant in the study area.

Prominent plant species mentioned during this study were Sesamum indicum, Dioclea sarmentosa, Clausena anisata, Anogeissus leiocarpus, Alafia barteri.

Various parts of plant especially leaves, roots, stem barks, seeds, fruits and whole plants were mentioned to be efficient in the treatment of menstrual disorders and postpartum health challenges among women in Abeokuta South Local Government Area. However, Leaves were reported to be the most frequently used plant parts; this could be an indication that leaves are sites where more phytochemicals are synthesized via photosynthesis (Odutuga et al., 2010: Kadiri et al., 2014). Although plants were reported to be used in various forms such as dry, fresh and in combination of both but preference was given to the use of the plants when they are freshly collected. This could be an indication that medicinal contents of plants are readily available when the plants are freshly collected and used immediately as some of the medicinal metabolites of some of these plants are volatile. This corroborates the findings of Devi Prasad et al., 2014 who reported that fresh plant material was used to prepare remedies as mixtures of multiple ingredients from different plants.

Some of the recipes are prepared from a single plant specie, for example, Momordica charantia, Ceiba petandra, Cissus quadrangularis, Phyllanthus muellerianus while Capsicum frutescens, Afromamomum melegueta, Aframomum melegueta, Capsicum frutescens Pterocarpus osun, Sorghum bicolour, Lannea egregia Daniellia oliveri, Lannea egregia, Anogeissus leiocarpus and Piliostigma thonningii are used in combinations with other common plants, as the combination of the plants was claimed to have higher medicinal effects on the treatment of the diseases although the respondents could not provide scientific reason for this findings. However, this observation could be justified with findings of Kadiri et al., 2013 who reported that malaria herbal preparations work better if two or more plants parts are prepared, as the phytochemicals of one part enhance the activities of the other.

Method of preparation varies; decoctions and grinding into powder are the most frequently used methods, this may be due to the type of plant part used and the choice of solvent in preparing the herbal remedies. Most of the herbal preparations collated during this study were reported to be used by oral application and bathing. It was found out from the practitioners that the plants administered as decoctions were characterized with tough leaves, bark and even the roots. They therefore had to be boiled longer to soften their parts before being administered. This method however, may not be most appropriate since the subjection of the plant material to high temperature is highly likely to alter the chemical composition of the plant, especially the very volatile ones as reported in past research studies (Okach, 2013; Jeruto et al., 2011).

Infusion method was used to administer the herbs that had delicate soft parts where plant leaves or the whole plant were dipped in hot water and left for some time for the active ingredients to be extracted. The plants that were macerated were found also to have highly soluble chemical components that would easily dissolve in cold water when left overnight in a covered container, as has also been reported in the past (Okach, 2013).

The result of this survey showed that majority (96\%) of the respondents claimed no occurrence of side effects following patients' use of herbal preparations. It could be that, the herbal preparations do not have any undesirable effects when used.

Quantitative phytochamicals studies on the most frequently mentioned plants helped to understand the pharmacological actions of the active compounds in these plants.

It has been proved scientifically that environmental factors and agricultural practices may significantly influence productivity, oil content and chemical composition of plant species (Daniel et al., 2011). The phytochemicals analysis conducted revealed that all the plant species collected contained tannins and saponins, alkaloids, saponnins and phenol.

The observation that plants such as Sesamum indicum Pterocarpus osun, Plumbago zeylanica, Piper guineensis, Sulphur, Citrus medica reported in this study were used for the management of these diseases is a clear indication that such plants have active medicinal values. The various medicinal properties exhibited by the various phytochemicals are useful in the treatment of most common ailments more importantly menstrual disorder and possible health challenges women do experience at postpartum.

These phytochemicals according to the literatures help in the purification of blood and excessive vaginal discharge and fight uterus infection (Tarun Chandra Taid et al 2014). Also, Shadma and Naheed, 2014 reported that plants were used to treat menstruation problems, for infant care and postpartum recovery. In the similar trend, Tarun Chandra Taid et al 2014 reported that herbal preparation during and after pregnancy help fight uterus infection, restore menstruation irregularity, stop excessive bleeding during menstruation, heal wound caused by contraction in relation of pelvic girdle experienced during parturition and act as energy stimulants which provide stamina to women after giving birth. Findings of Owu, 2004 revealed that the expectorant property of medicinal plants is attributed to the presence of saponins due to their ability to produce form which act as cleansing agent.

The observation that the metabolites of the plants were found both in the leaves and roots of these plants is a clear indication that the two parts under consideration are rich in phytochemicals that are active in the treatment of these aiments. The study also revealed that some plants collated are characterized by phytochemical contents active in the treatment of menstrual disorders and after-childbirth problems. Ikeyi and Omeh, 2014 in their work on a review of the Ethnotherapeutics of medicinal plants used in traditional/alternative medicinal practice in Eastern Nigeria reported that plants collated were observed to 
contain potential chemo preventive agents, viz: Alkaloids, Glycosides, and Saponins, Phytosterol, flavonoids and phenol compounds in varying quantities. The observation of tannins in these plants could have oxidation inhibiting activity and confer good flavour on leaves (Nwauzoma, et al., 2013). The presence of saponin is well reported in plants (Belewu et al., 2009), where they served as expectorants and emulsifying agents. Saponins are glycosides with distinctive foaming characteristics (Nwauzoma, et al., 2013).

\section{CONCLUSION}

\section{REFERENCES}

1. Daniel, V. N, Daniang, I. E, Nimye, N. D.. Phytochemical analysis and mineral elemental composition of Ocimum basilicum obtained in Jos Metropolis, Plateau state, Nigeria. International Journal of Engineering and Technology, 2011, 11(6): 161-165.

2. Devi Prasad1, A.G Shyma, T. B., and Raghavendra, M. P. Traditional herbal remedies used for management of Reproductive disorders in wayanad district, kerala, International journal of research in pharmacy and chemistryijrpc, 2014, 4(2), 333-341

3. Hajaratu, U. S., Nkiruka, A., Adebiyi, G .A, Ijeoma, C .O, Austin, O. O., Solomon, A., Nosa, E .E., Afolabi, I .Y., Abdulsalam, M.Age at menarche and prevalence of menstrual abnormalities among adolescents in Zaria, northern Nigeria, Annals of Nigerian Medicine, 2014, Vol : 7 ( 2): 66-70

4. Ikeyi, P. A and Omeh, N.Y. 2014 . A review of the ethnotherapeutics of medicinal plants used in traditional/alternative medicinal practice in eastern Nigeria, Int.J.Curr.Microbiol.App.Sci (2014) 3(1): 675-683

5. Jeruto, P.M. C, Lukhoba, C, O. G.. Phytochemical composition of some medicinal plants used by the Nandi of South Nandi district, Kenya. Journal of Plant and Animal, 2011, Sciences 9(3):1201-1210.

6. Kadiri, M Ojewumi, A.W., Adebiyi, D.T., Yahaya, M.,. Bala S.A. Ethno-phytotherapy of plants used for managing diarrhea in Abeokuta, Oguin State, Nigeria. International Journal of Green and Herbal Chemistry, 2014, 3(3): 1307-1319.

7. Kadiri, M., Ojewumi, A.W., and Adegboye, O.O. Folk use of herbal plants used in the treatment of malaria fever in Abeokuta North Local Government, Ogun, State. Nigeria. Direct Research Journal of Health and Pharmacology (DRJHP), 2013, 1 (2): 10-19

8. Nitta, T., Arai, T., Takamatsu, H., Inatomi, Y., Murata, H., Iinuma, M., Tanaka, T., Ito, T., Asai, F., Ibrahim, I., Nakanishi, T. and Watabe, K. y. Antibacterial activity of extracts prepared from tropical and subtropical plant sonmethicillin-resistant staphylococcus aureus. Journal of Health Science, 2002, 48,273-276.

9. Nwauzoma, A. B. And Dawari, Songo L. studies on thephytochemical properties and proximate analysis of piper
It could be concluded that although few people can still afford the financial demands of modern drugs for simple and complicated diseases yet majority of people in Abeokuta South Local Government depend on medicinal plants for the treatment of menstrual disorders and after childbirth problems. Also, considering the undisputed role played by these medicinal plants in the modern day world in the health care system of rural populace, it is of outmost importance that these plants are cultivated, propagated and protected. Younger generation should be encouraged towards tapping wealth from what they regarded as wastes. umbellatum (linn) from nigeria, American Journal of Research Communication, 2013, Vol 1(7)

10. Odutuga, A.A., Dairo, J.O., Minari, J.B. and Bamisaye, F.A. (Antidiabetic effect of Morinda lucida stembark extract on alloxan-induced diabetic rat. Journal of Pharmacology, 2010, 4 (3): 78-82.

11. Ojewumi, A.W, and Kadiri, M. Phytochemical screening and anti-diabetic properties of Terminalia schimperiana Leaves on rats, International Journal of Green and Herbal Chemistry, 2014, 3 (4):1679-1689.

12. Okach D.O., Nyunja A.R.O., Opande G. Phytochemical screening of some wild plants from Lamiaceae and their role in traditional medicine in Uriri District-Kenya, International Journal of Herbal Medicine, 2013, 1 (5): 135-143

13. Okwu, D. E. 2004. Phytochemical and vitamin content of indigenous spices of South Eastern Nigeria. Journal of Sustainable Agricultural Environment, 2014, 6:140-147.

14. Olajide, O. Steps towards sustainable natural forest management for non- timber forest production in Nigeria. Proceeding of Annual conference of the Forest Association of Nigeria, Cross River State. 6th-11th March, 2013.

15. Ramana, M. V. Ethnobotanical and ethnoveterinary plants from Boath, Adilabad District and Thraprudesh, India. Ethnobotanical leaflets. 2008, 12: 391-400.

16. Shadma Shahin and Naheed Ahmad. Herbs used during pregnancy and post partum in a group of women in patna district inter. J. of Phytotherapy, Sciences 9(3):1201-1210. Vol 4 ( 2): 58-62.

17. Sheldon, J.W., Balick M. J., Laird, S. A. Medicinal plants: can utilization and conservation co-exist? Advances in Economic Botany, 1997, 12: 1-104.

18. Tarun Chandra Taid, Ratul Chandra, R and Jogen, C. K. A study on the medicinal plants used by the local traditional healers of Dhemaji district, Assam, India for curing reproductive health related disorders, Advances in Applied Science Research, 5(1):296-301, 2014.

19. Ukaegbe, C.I Iteke, O.C Bakare, MO Agbata, AT. Postpartum Depression Among Igbo Women In An Urban Mission Hospital, South East Nigeria, Ebonyi Medical Journal, 2012, $11: 1-2$ 